\title{
Una aplicación del AHP para la obtención de las preferencias de los estudiantes sobre las competencias en educación superior. An application of AHP to obtain the students' preferences over the competences in higher education.
}

\author{
Jesús Palomo ${ }^{1}$, Mónica de Castro ${ }^{2}$, Pilar Laguna ${ }^{1}$, Concepción de la Fuente ${ }^{1}$ \\ jesus.palomo@urjc.es, monica.decastro@urjc.es, pilar.laguna@urjc.es.concepcion.delafuente@urjc.es \\ ${ }^{1}$ Departamento de Economía de la Empresa \\ Universidad Rey Juan Carlos \\ Madrid, España \\ ${ }^{2}$ Rectorado \\ Universidad Rey Juan Carlos \\ Madrid, España
}

\begin{abstract}
Resumen- El marco europeo actual de enseñanza superior centra sus objetivos de aprendizaje en la adquisición de competencias. Aunque se ha estudiado ampliamente la evaluación de competencias en este contexto, hasta la fecha no se han desarrollado metodologías multicriterio que incorporen las preferencias de los estudiantes sobre las competencias. En este trabajo se presenta una aplicación del análisis de jerarquías analíticas para la obtención de las preferencias grupales de los estudiantes sobre la importancia de las competencias de una asignatura. El análisis empírico se ha realizado con estudiantes de cuatro titulaciones distintas de la Universidad Rey Juan Carlos que comparten la asignatura Dirección Financiera II. Las preferencias se han obtenido sobre cinco competencias generales y dos competencias específicas de esta asignatura.
\end{abstract}

\section{Palabras clave: competencias, AHP, participación}

Abstract- The current European framework for higher education focuses the learning objectives on the acquisition of skills or competencies. Although the evaluation of competences in this context has been extensively studied, no multi-criterion methodologies, that incorporate students' preferences on competencies, have been developed so far. This paper presents an application of analytic hierarchy process to obtain group preferences of the students on the importance of the competences in a course. The empirical analysis has been carried out with students of four different degrees from the Rey Juan Carlos University who share the subject Financial Management II. The preferences have been obtained on five general competences and two specific competences of this subject.

\section{Keywords: competences, AHP, participation}

\section{INTRODUCCIÓN}

El Espacio Europeo de Educación Superior (EEES) se centra en la adquisición de competencias como soporte del aprendizaje. En los últimos años se han hecho verdaderos esfuerzos en definir metodologías de valoración de los sistemas educativos basados en competencias (Bergsmann et al., 2015). Sin embargo, aunque numerosos estudios muestran la necesidad creciente de integrar las preferencias de los estudiantes en el diseño de los planes de estudio (Iahad et al, 2013; Lelis, 2017; Martín-Peña et al., 2012), esta integración no ha sido suficiente. En este sentido, la mayoría de los trabajos que evalúan la modalidad presencial utilizan técnicas participativas basadas en la comunicación personal entre profesores y alumnos (Tsien y Tsui, 2007; Yakovleva y Yakovlev, 2014). Esto contrasta con la evaluación del elearning, modalidad de enseñanza que más trabajos ha concentrado en los últimos años, que aplica métodos muy estructurados de análisis (Hung y Chou, 2015; Kearns, 2016; Ozkan y Koseler, 2009; Wu et al., 2010).

La heterogeneidad del alumnado y el creciente tamaño de los grupos hace que la gestión de la información resulte cada vez más difícil y sea necesario recurrir a métodos muy estructurados para recoger las valoraciones de todos los agentes implicados en el proceso educativo (Sander, 2000). En este sentido, el análisis de jerarquías analíticas (Analytic Hierarchy Process AHP en inglés) no ha sido ampliamente utilizado en este contexto, a pesar de que este tipo de análisis resulta especialmente adecuado para integrar percepciones individuales en entornos complejos con un gran número de elementos que evaluar (Vaidya y Kumar, 2006). De hecho, el AHP únicamente se ha utilizado para evaluar el rendimiento de las herramientas de e-learning (Chao y Cheng, 2009; Lin, 2010; Lin, T. C., Ho, H. P., \& Chang, C. T, 2014; Shee y Wang, 2008), no habiéndose usado en entornos presenciales. Hasta la fecha, los estudios que se han ocupado de analizar la satisfacción de los estudiantes sobre el logro de los objetivos del proceso de enseñanza-aprendizaje se han realizado sobre planes de estudio previamente definidos. Sin embargo, para una planificación eficaz de las políticas públicas resulta esencial incorporar la participación de los agentes implicados desde las primeras etapas del proceso (Nordström, E. M., Eriksson, L. O., \& Öhman, K., 2010). En este trabajo se presenta una aplicación de una metodología multi-criterio basada en las jerarquías analíticas para recoger la percepción del conjunto de estudiantes sobre la importancia de las competencias asociadas a una asignatura. Esta metodología permite recoger de manera rigurosa y estructurada las percepciones de los estudiantes individuales sobre la importancia de las competencias de una asignatura y proporcionar una valoración conjunta. La información grupal obtenida puede resultar muy útil para diseñar los programas docentes de enseñanza superior y desarrollar modelos 
participativos considerando las preferencias de uno de los principales agentes implicados: los estudiantes.

\section{CONTEXTO}

La preocupación por mejorar la calidad de los sistemas de enseñanza en el EEES es cada vez mayor (Bergsmann, E., Schultes, M. T., Winter, P., Schober, B., \& Spiel, C., 2015).

Paralelamente, en este contexto democrático y complejo se requieren modelos de evaluación participativos, donde todos los agentes implicados participen en los procesos de toma de decisiones, y que sean capaces de gestionar eficientemente la información. De hecho, las directrices de calidad del modelo europeo de educación superior recomiendan tener en cuenta dos cuestiones fundamentales: (1) el desarrollo de sistemas de evaluación capaces de recoger la participación y autonomía de los estudiantes en el proceso de aprendizaje y (2) la gestión de la información en los procesos de toma de decisiones (EC, 2015).

\section{A. La participación de los estudiantes en el diseño de los planes de estudio.}

El EEES es un entorno complejo donde interviene un gran número de agentes y democrático, en el que se incentiva, cada vez, más la participación de estudiantes y docentes en el proceso de enseñanza-aprendizaje (Laal, M., KhattamiKermanshahi, Z., \& Laal, M., 2014). Se observa una creciente preocupación por incorporar a los estudiantes en los procesos de toma de decisiones que intervienen a lo largo del proceso educativo, tanto en el desarrollo del proceso de enseñanzaaprendizaje en sí, como en su diseño, planificación y evaluación.

Un gran número de trabajos se centran en el desarrollo de modelos de enseñanza en los que los estudiantes participan activamente en las clases (Iahad, N. A., Mirabolghasemi, M., Mustaffa, N. H., Latif, M. S. A., \& Buntat, Y. , 2013; Yakovleva y Yakovlev, 2014; van Wyk, 2013; Montalvo y Palomo, 2010; Palomo y Montalvo, 2011; Figueroa-Domecq, C, de la Orden, C., \& Palomo, J. , 2016; de la Orden, C., Palomo, J., \& Figueroa-Domecq, C., 2017), mientras que otros estudios incorporan la participación de los estudiantes en el proceso de evaluación, valorando la adquisición de competencias (Bergsmann et al., 2015; Martín-Peña, M. L., Díaz-Garrido, E., \& del Barrio Izquierdo, L., 2012).

Sin embargo, las percepciones de los estudiantes no suelen ser consideradas en la fase de diseño o adaptación de los planes de estudio. Varios trabajos destacan la importancia de considerar de manera formal las expectativas de los estudiantes en el diseño de modelos educativos de enseñanza superior para mejorar la eficacia del entorno de aprendizaje (Sander, P., Stevenson, K., King, M., \& Coates, D., 2000; Voss, R., Gruber, T., \& Szmigin, I., 2007). Se ha observado que el conocimiento por parte del docente de las expectativas del estudiante permite mejorar el rendimiento del proceso de enseñanza-aprendizaje (Sander, 2005). Sin embargo, recoger las percepciones de los estudiantes cuando los grupos son grandes y heterogéneos resulta complicado y es necesario usar métodos formales muy estructurados. Esta diversidad cada vez mayor del alumnado requiere prestar especial atención a la metodología de agregación de las valoraciones individuales. Algunas técnicas de análisis multi-criterio, como el AHP se han utilizado poco en este ámbito, pero son capaces de integrar las preferencias de varios decisores de manera rigurosa, objetiva y estructurada, en los procesos toma de decisiones (Saaty, 2001).

\section{B. El análisis de jerarquías analíticas para la valoración de competencias.}

El AHP es un método multi-criterio que permite recoger valoraciones subjetivas y cuantificar los trade-offs entre pares de criterios intangibles (Saaty, 2001). Por este motivo, su área de aplicación es muy amplia y se ha utilizado con éxito para resolver una amplia variedad de problemas de toma de decisiones en las empresas y en las administraciones públicas donde se requiere el consenso de un grupo (Bryson, 1996). Sin embargo, para evaluar la educación superior, en particular la modalidad presencial, no se ha aplicado. De hecho, únicamente se ha usado para evaluar entornos de e-learning. Shee y Wang (2008) y Lin et al. (2014) utilizan AHP para evaluar la satisfacción de los usuarios de una plataforma de elearning. Chao y Chen (2009) combinan AHP con funciones Fuzzy para evaluar un entorno de e-learning. Recientemente, de Castro et al. (2017) han usado AHP para evaluar la capacidad de algunas herramientas de e-learning para la adquisición de una competencia por estudiantes de empresa.

Las principales críticas que ha recibido este método son asociadas a las inconsistencias de algunas respuestas y al número limitado de criterios que se pueden usar para que el método resulte operativo. El primer inconveniente se ha resuelto usando un modelo de programación por metas como se indica en la Sección 3. Respecto a las limitaciones en el número de criterios, como se muestra en la Sección 4, es posible seleccionar un número de competencias suficiente para obtener resultados robustos y mantener la operatividad del método.

Este artículo, a través del AHP, se centra en dos cuestiones clave que resaltan las directrices europeas: incorporar la participación de los estudiantes para el diseño de planes docentes y gestionar la información de manera rigurosa. La metodología propuesta puede resultar muy útil para las instituciones universitarias europeas de educación superior que quieran mejorar la participación en sus programas docentes siguiendo las recomendaciones de calidad del EEES.

Como caso de estudio empírico, se han recogido las percepciones de los estudiantes sobre la importancia de las competencias asociadas a la asignatura Dirección Financiera II del Grado en Administración y Dirección de Empresas en la Universidad Rey Juan Carlos.

\section{DESCRIPCIÓN}

El AHP considera las preferencias o valoraciones individuales a través de juicios de valor sobre la importancia relativa de los criterios y las alternativas tomadas por pares. Esta metodología se basa en tres etapas: modelización, valoración, y priorización y síntesis (Saaty, 2001). En la etapa de modelización se identifican los criterios y las alternativas del problema, y se organizan según una estructura jerárquica. En la etapa de valoración, se recogen las preferencias, gustos y deseos de los actores mediante los juicios incluidos en las denominadas matrices de comparaciones pareadas (Moreno- 
Jiménez, 2002). Por último, en la etapa de priorización y síntesis se obtienen las prioridades locales, globales y totales.

Para obtener una estructura jerárquica de las 7 competencias a valorar, a cada estudiante se le ofrece la opción de expresar su intensidad de preferencia, en una escala de 1-9 puntos de tipo Saaty (2001), sobre un par de atributos. Como se describe en la Tabla 1, si dos atributos tienen la misma importancia, se asigna una puntuación de 1 a esa comparación, mientras que la puntuación 9 indica la importancia absoluta de un criterio sobre el otro. Una vez recogidas las preferencias de los decisores, el AHP utiliza el método del auto-vector principal por la derecha para obtener las prioridades locales; el principio de composición jerárquico para calcular las prioridades globales y una forma lineal multiaditiva para obtener las prioridades totales (Moreno-Jiménez, 2002).

Tabla 1

Escala de importancia en una encuesta de tipo Saaty

\begin{tabular}{|c|c|c|}
\hline $\begin{array}{l}\text { Escala } \\
\text { numérica }\end{array}$ & Escala Verbal & Descripción \\
\hline 1 & Igual importancia & $\begin{array}{l}\text { Los dos elementos } \\
\text { contribuyen igualmente } \\
\text { a la propiedad o criterio }\end{array}$ \\
\hline 3 & $\begin{array}{l}\text { Moderadamente más } \\
\text { importante un } \\
\text { elemento que el otro }\end{array}$ & \begin{tabular}{lrr} 
El juicio & y & la \\
experiencia & \multicolumn{2}{r}{ previa } \\
favorecen & a & un \\
elemento & respecto & al \\
otro & &
\end{tabular} \\
\hline 5 & $\begin{array}{l}\text { Fuertemente más } \\
\text { importante un } \\
\text { elemento que el otro }\end{array}$ & $\begin{array}{l}\text { El juicio y la } \\
\text { experiencia previa } \\
\text { favorecen fuertemente a } \\
\text { un elemento respecto al } \\
\text { otro }\end{array}$ \\
\hline 7 & $\begin{array}{l}\text { Mucho más } \\
\text { importante un } \\
\text { elemento que el otro }\end{array}$ & $\begin{array}{l}\text { Un elemento domina } \\
\text { fuertemente }\end{array}$ \\
\hline 9 & $\begin{array}{l}\text { Extremadamente más } \\
\text { importante un } \\
\text { elemento que el otro }\end{array}$ & $\begin{array}{l}\text { Un elemento domina al } \\
\text { otro con el mayor orden } \\
\text { de magnitud posible }\end{array}$ \\
\hline
\end{tabular}

Las ponderaciones determinan la importancia relativa de cada atributo respecto del resto, y constituyen la base sobre la cual definir una ordenación de las alternativas del problema de decisión (Saaty, 2001). Para el tratamiento de las inconsistencias se propone el modelo de González-Pachón y Romero (2004).

En el contexto descrito en este artículo, la metodología se aplica comparando pares de competencias de una asignatura para definir el ranking de importancia o valoración conjunta que tiene el grupo de estudiantes sobre dichas competencias.

\section{Resultados}

Las competencias definidas en la asignatura Dirección Financiera II del Grado en ADE de la Universidad Rey Juan Carlos se subdividen en: 5 competencias generales y 2 competencias específicas. Las competencias generales son:

CG1-Análisis y síntesis.

CG2-Gestión de la información.
CG3-Toma de decisiones.

CG4-Capacidad para aprender.

CG5-Capacidad para trabajar autónomamente.

Las competencias específicas son:

CE1-Comprensión de operaciones financieras en el ámbito empresarial.

CE2-Resolver problemas de valoración financiera (decisiones financieras e inversión empresarial).

La modelización del problema se ha definido en base a la estructura jerárquica que se representa en la Figura 1. El objetivo corresponde a la valoración de la importancia de las competencias y los criterios son las 7 competencias a valorar.

Istructura Jerárquica

Objetivo

Criterios

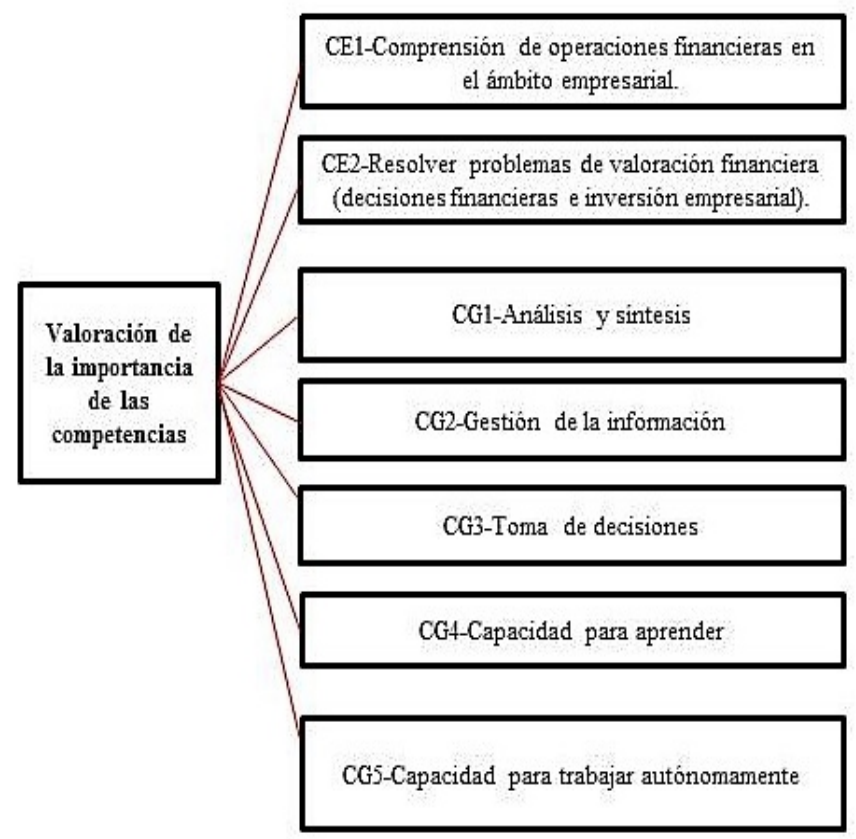

Figura 1: Estructura jerárquica de las competencias de Dirección Financiera II

Para obtener la valoración de los estudiantes se ha realizado una encuesta de tipo Saaty (Saaty, 2001) en formato online a cuatro grupos de estudiantes de Dirección Financiera II.

En total se han recogido 210 encuestas válidas, de las cuales 140 han obtenido un índice de inconsistencia inferior a 0,40. Las respuestas inconsistentes se han tratado con un modelo de programación por metas (González-Pachón y Romero, 2004), obteniendo matrices lo más parecidas posibles a las matrices primarias, pero sin inconsistencia. Con este método se ha conseguido mejorar la inconsistencia de 67 matrices que inicialmente presentaban un índice de inconsistencia superior a 0,40. En la Tabla 2 se muestra el número de valoraciones primarias, el número de valoraciones primarias con un índice de inconsistencia inferior a 0,40 y el número de valoraciones útiles después de corregir las inconsistencias. 
Tabla 2

Valoraciones primarias totales, primarias válidas y válidas tras la corrección de inconsistencias

\begin{tabular}{lll}
\hline $\begin{array}{l}\text { Valoraciones } \\
\text { primarias }\end{array}$ & $\begin{array}{l}\text { Valoraciones } \\
\text { con IC }<\mathbf{0 . 4 0}\end{array}$ & $\begin{array}{l}\text { Valoraciones } \\
\text { útiles tras la } \\
\text { corrección de } \\
\text { inconsistencias }\end{array}$ \\
\hline 210 & 140 & 207
\end{tabular}

Los resultados muestran las competencias CE1 (Comprensión de operaciones financieras en el ámbito empresarial), y CG3 (Toma de decisiones) como las más importantes para los estudiantes, seguidas de CE2 (resolver problemas de valoración financiera) y CG4 (capacidad de aprender). Por otro lado, las competencias CG2 (Gestión de la información), CG1 (Análisis y síntesis) y CG5 (Capacidad para trabajar autónomamente) han obtenido las valoraciones más bajas. Los pesos globales que han obtenido cada una de las competencias se muestran en la tabla 3.

Tabla 3

Prioridades globales de las competencias

\begin{tabular}{ll}
\hline Competencias & $\begin{array}{l}\text { Prioridades } \\
\text { Globales }\end{array}$ \\
\hline CE1 & 0.20 \\
CE2 & 0.16 \\
CG1 & 0.10 \\
CG2 & 0.11 \\
CG3 & 0.19 \\
CG4 & 0.14 \\
CG5 & 0.10 \\
\hline
\end{tabular}

Estos resultados indican que para los estudiantes del área de empresa, es más importante el enfoque más aplicado a un contexto real, como la comprensión de esta asignatura de finanzas en el entorno de la empresa, así como la capacidad para tomar decisiones, o para aprender. Las competencias vinculadas al contenido teórico del aprendizaje, como la organización de la información o el aprendizaje autónomo, resultan menos relevantes. La reducida importancia que han obtenido las competencias asociadas al aprendizaje autónomo y a las capacidades asociadas a la gestión de la información, frente a la importancia de las competencias más aplicadas en contextos empresariales en los que se precisa la interacción con otros agentes asociados a un entorno profesional real, sugiere que los estudiantes precisan un aprendizaje dirigido y aplicado, donde el rol del docente debería adquirir un mayor protagonismo y los recursos docentes deberían orientarse hacia actividades aplicadas, como casos de estudio y actividades en entornos profesionales.

Esta información es útil integrar las preferencias de los estudiantes en los planes de estudio, incorporando en la guía de la asignatura recursos docentes adecuados a este orden de prioridades.

Los resultados muestran igualmente que el AHP junto con la programación por metas pueden resultar herramientas eficaces para recoger información rigurosa y útil para la evaluación de la enseñanza superior, considerando la participación de los estudiantes en los procesos.

\section{CONCLUSIONES}

Los resultados muestran que el análisis AHP puede resultar una herramienta eficaz para recoger las percepciones de los estudiantes y proporcionar valoraciones grupales para la definición o revisión de programas en educación superior. En el caso de estudio presentado, esta metodología ha permitido obtener las valoraciones de los estudiantes de titulaciones del área de empresa sobre la asignatura Dirección Financiera II y se puede concluir que los estudiantes consideran más importantes las competencias vinculadas a un aprendizaje aplicado de la asignatura, como las competencias relacionadas con la resolución de problemas y con la comprensión de las operaciones financieras en un contexto real. La metodología empleada en este caso de estudio ha permitido un tratamiento riguroso de la información, tanto en la recogida y proceso de las valoraciones individuales como en su agregación para obtener una única valoración conjunta.

La metodología propuesta puede servir de base para el desarrollo de modelos participativos en el marco europeo de enseñanza que se encuentren alineados con las preferencias de los estudiantes. Sería muy recomendable dirigir futuras líneas de investigación a la aplicación de otras técnicas multi-criterio que permitan desarrollar modelos participativos para el diseño de políticas educativas de calidad siguiendo las recomendaciones de los convenios internacionales de educación superior.

\section{REFERENCIAS}

Bergsmann, E., Schultes, M. T., Winter, P., Schober, B., \& Spiel, C. (2015). Evaluation of competence-based teaching in higher education: From theory to practice. Evaluation and program planning, 52, 1-9.

Bryson, N. (1996). Group decision-making and the analytic hierarchy process: Exploring the consensus-relevant information content. Computers \& Operations Research, 23(1), 27-35.

Chao, R. J., \& Chen, Y. H. (2009). Evaluation of the criteria and effectiveness of distance e-learning with consistent fuzzy preference relations. Expert Systems with Applications, 36(7), 10657-10662.

Colace, F., de Santo, M. and Pietrosanto, A. (2006) Evaluation models for e-learning platform: an AHP approach. 36th Annual Frontiers in Education (FIE 2006). San Diego, Oct.28-31, 2006 .

de Castro, M., de la Fuente-Cabrero, C., \& Sánchez, M. D. P. L. (2017). Assessment of Autonomous Learning Skill Through Multi-criteria Analysis for Online ADE Students in Moodle. In Entrepreneurial Universities (pp. 197-213). Springer International Publishing.

de la Orden, C., Palomo, J., \& Figueroa-Domecq, C. (2017) Improving learning by motivating students to read the news using ICT. Global Journal of Business, Economics and Management, 7(1), 89-98.

European Comission (2015) Standars and guidelines for quality assurance in the European Higher Education Area (ESG). 
Figueroa-Domecq, C, de la Orden, C., \& Palomo, J. (2016) Internet como Fuente de Contenidos Prácticos Complementarios a los Reglados en las Asignaturas de Grado. En La innovación docente con TIC como instrumento de transformación (pp. 225-235). Dykinson. ISBN 9788490859742

González-Pachón, J., \& Romero, C. (2004). A method for dealing with inconsistencies in pairwise comparisons. European Journal of Operational Research, 158(2), 351361.

Hung, M. L., \& Chou, C. (2015). Students' perceptions of instructors' roles in blended and online learning environments: A comparative study. Computers \& Education, 81, 315-325.

Iahad, N. A., Mirabolghasemi, M., Mustaffa, N. H., Latif, M. S. A., \& Buntat, Y. (2013). Student perception of using case study as a teaching method. Procedia-Social and Behavioral Sciences, 93, 2200-2204.

Kearns, L. R. (2016). The experience of teaching online and its impact on faculty innovation across delivery methods. The Internet and Higher Education, 31, 71-78.

Laal, M., Khattami-Kermanshahi, Z., \& Laal, M. (2014). Teaching and education; collaborative style. ProcediaSocial and Behavioral Sciences, 116, 4057-4061.

Lelis, C. (2017). Participation ahead: Perceptions of master degree students on reciprocal peer learning activities. Journal of Learning Design, 10(2), 14-24.

Lin, H. F. (2010). An application of fuzzy AHP for evaluating course website quality. Computers \& Education, 54(4), 877-888.

Lin, T. C., Ho, H. P., \& Chang, C. T. (2014). Evaluation Model for Applying an E-Learning System in a Course: An Analytic Hierarchy Process-Multi-Choice Goal Programming Approach. Journal of Educational Computing Research, 50(1), 135-157.

Martín-Peña, M. L., Díaz-Garrido, E., \& del Barrio Izquierdo, L. (2012). Metodología docente y evaluación por competencias: una experiencia en la materia Dirección de Producción. Investigaciones europeas de dirección y economía de la empresa, 18(3), 237-247.

Montalvo, S. \& Palomo, J. (2010) Bridging the gap between teaching and breaking news: A new approach based on ESHE and ICT. Procedia-Social and Behavioral Sciences, 9, 1423-1428.

Jiménez, J. M. M. (2002). El proceso analítico Jerárquico (AHP). Fundamentos, metodología y aplicaciones. Caballero, R. y Fernández, GM Toma de decisiones con criterios múltiples. RECT@. Revista Electrónica de Comunicaciones y Trabajos de ASEPUMA. Serie Monografías, (1), 21-53.
Nordström, E. M., Eriksson, L. O., \& Öhman, K. (2010). Integrating multiple criteria decision analysis in participatory forest planning: Experience from a case study in northern Sweden. Forest Policy and Economics, 12(8), 562-574.

Ozkan, S., \& Koseler, R. (2009). Multi-dimensional students' evaluation of e-learning systems in the higher education context: An empirical investigation. Computers \& Education, 53(4), 1285-1296.

Palomo, J. \& Montalvo, S. (2011). Una plataforma internacional de apoyo a la docencia basada en noticias Arbor, 187(3), 249-253.

Saaty, T. L. (2001). Fundamentals of the analytic hierarchy process. In The analytic hierarchy process in natural resource and environmental decision making (pp. 1535). Springer Netherlands.

Sander, P., Stevenson, K., King, M., \& Coates, D. (2000). University students' expectations of teaching. Studies in Higher education, 25(3), 309-323.

Sander, P. (2005). La investigación sobre nuestros alumnos, en pro de una mayor eficacia en la enseñanza universitaria. Electronic Journal of Research in Educational Psychology, 3(1), 113-130.

Shee, D. Y., \& Wang, Y. S. (2008). Multi-criteria evaluation of the web-based e-learning system: A methodology based on learner satisfaction and its applications. Computers \& Education, 50(3), 894-905.

Tsien, T. B., \& Tsui, M. S. (2007). A participative learning and teaching model: The partnership of students and teachers in practice teaching. Social Work Education, 26(4), 348-358.

Vaidya, O. S., \& Kumar, S. (2006). Analytic hierarchy process: An overview of applications. European Journal of operational research, 169(1), 1-29.

Voss, R., Gruber, T., \& Szmigin, I. (2007). Service quality in higher education: The role of student expectations. Journal of Business Research, 60(9), 949-959.

Wu, J. H., Tennyson, R. D., \& Hsia, T. L. (2010). A study of student satisfaction in a blended e-learning system environment. Computers \& Education, 55(1), 155-164.

Van Wyk, M. M. (2013). The use of economic games as a participative teaching strategy to enhance student Learning. Journal of social science, 35(20), 125-133.

Yakovleva, N. O., \& Yakovlev, E. V. (2014). Interactive teaching methods in contemporary higher education. Pacific Science Review, 16(2), 75-80. 